\title{
Working better together: joint leadership development for doctors and managers
}

\section{Nicola Kelly}

Birmingham Children's Hospital

\begin{abstract}
Traditionally, there have been tensions between frontline healthcare professionals and managers, with well-known stereotypes of difficult consultants and pen-pushing managers. Many junior doctors have limited management experience and have often never even met a manager prior to taking on a consultant role.
\end{abstract}

Based on a successful programme pioneered by Dr Robert Klaber (Imperial, London) we have set-up an innovative scheme for Birmingham Children's Hospital, pairing junior doctors and managers to learn and work together. Our aim was to cultivate positive attitudes and understanding between the two groups, break down inter-professional barriers, and to provide practical leadership experience and education.

We recruited 60 managers and doctors to participate in shadowing, conversation, and quality improvement projects. Thought-provoking online materials, blogs, socials, and popular monthly workshops consisting of patient-focused debate and discussion around key leadership themes, have helped to support learning and cement shared values.

Formal evaluation has demonstrated an improvement in how participants perceive their knowledge and ability based on key NHS Leadership Framework competencies. Participant feedback has been extremely positive, and everyone plans to continue to incorporate Paired Learning into their continuing professional development.

We are now embedding Paired Learning in the on-going educational programme offered at Birmingham Children's Hospital, whilst looking at extending the scheme to include different professional groups and other trusts across the region and nationally.

\section{Problem}

Historically in the NHS, clinical professionals and managers have struggled to find ways to work effectively together, with some doctors not even meeting a manager prior to being a consultant.

The NHS Leadership Framework is applicable to all healthcare professionals, and outlines how we can each incorporate leadership development throughout our careers. Hospital trusts and deaneries need to find ways to provide management and leadership training opportunities for junior doctors, nurses, allied healthcare professionals, and managers in locally based programmes.

\section{Background}

In today's health service, good team working clinical professionals and managers has never been more important. Speaking about doctors and managers in his 2014 New Year message (1), Dr Mark Porter (BMA Chair) states, "We can no longer afford to have two tribes, because two tribes bring half the benefit to patients". Stronger leadership and shared values are to be major priorities for NHS teams aiming to improve patient outcomes and the culture within organisations, as highlighted by the recent Francis report. Both the Francis (2) and Darzi (3) reform reports stress the importance of frontline staff becoming more engaged in NHS leadership and managing the improvement of patient services. One American doctor (4) claims that clinician engagement is a key sign of a better performing hospital.

London Paediatrician, Dr Bob Klaber, noticed how managers and junior doctors in his trust rarely spoke and knew little of each other's work. To address this issue he launched the successful London Deanery Paired Learning Scheme (5), which links senior registrars with managers to share expertise in improving services. Inspired by this approach, we wanted to develop a similar scheme for staff at Birmingham Children's hospital.

\section{Baseline measurement}

After recruiting participants for our scheme (see 'Project design'), we distributed pre-programme evaluation questionnaires. The questionnaire asked participants to rate on a 5-point scale their own knowledge and ability against key competencies from the NHS Leadership Framework. The results are shown (see supplementary file) alongside the post-programme results for comparison. We also used the questionnaires to discover what participants would like to get out of the scheme, and used this information to plan our workshops and other events.

\section{Design}


Lydia Salice, a service manager and I formed a 'pilot' pair in December 2013, teaming up to establish the Paired Learning scheme at Birmingham Children's Hospital as part of our NHS Change Day 2014 pledge.

We identified target groups for our scheme as junior doctors of all grades, and non-clinical managers as we felt it was these groups that would benefit the most from the scheme and provide the greatest contrast of experience. For the pilot scheme we decided that we would offer the opportunity to all members of those groups to participate, and use a pre-scheme questionnaire to guide our pairings.

We were aware that doctors and managers are very busy people with heavy work schedules, and did not want anyone to be deterred from participating due to concerns about excessive time commitment. We therefore offered a range of different activities, allowing participants to choose the depth and extent of their involvement. All participants met and shadowed their partner, many attended the monthly workshops and socials, whilst some pairs undertook joint service improvement projects.

We secured financial support from our trust education and development team and senior deanery figures, which funded speaker's expenses, workshop resources, and refreshments. We decided to launch a 6-month pilot Paired Learning scheme and then re-evaluate our efforts.

\section{Strategy}

To start with, we reviewed the Imperial Paired Learning report, and discussed which aspects of their scheme we would like to recreate and any ideas we had of what we could do differently at Birmingham Children's Hospital. We formed a small focus group with other senior and junior doctors and managers, meeting to discuss our ideas and plans for implementing our scheme.

We consulted with key stakeholders and gained overwhelming support from senior clinicians and managers by presenting our proposals for the scheme at key leadership forums within the trust.

We then advertised the scheme through posters, meeting announcements, email, and word of mouth in order to recruit participants. 60 people expressed an interest in the scheme; an equal mix of doctors and managers. We also made a video explaining our project, which is available on YouTube (6) and which is shown to new doctors and managers at trust induction days. We asked our recruits to complete a pre-evaluation form, which provided a baseline measure of participant's leadership competency, whilst informing us of key areas participants would like to see covered in workshops.

Our focus group then held a 'matchmaking' meeting, to create our pairings based on interests expressed in the pre-evaluation. Pairs met for the first time at our launch event in January 2014, at which we explained to them how they could gain insight into each other's roles and perspectives through dialogue and workplace shadowing
We also encouraged pairs to consider taking on a joint project to improve or develop a service that would enhance patient care.

We held regular monthly workshops to enable debate and discussion around key topics identified through pre-scheme questionnaires and participant consultation, such as Leadership Style and Managing Change. Speakers included our CEO SarahJane Marsh and NHS commentator Roy Lilley. We met with education team managers to source speakers and funding to make our workshops successful.

After 6 months of running the scheme, we re-evaluated by circulating a repeat questionnaire, and listening to feedback from participants at our Paired Learning celebration event, at which pairs shared with each other and guests from the trust and deanery what they had learned and achieved together.

\section{Results}

We evaluated our programme by comparing responses to a questionnaire where participants rated themselves on a 5-point scale based on NHS Leadership Framework competencies. Competency areas included working in partnership with managers/doctors, leading quality improvement initiatives, and understanding management/clinical structures and hierarchies.

The evaluation demonstrated improvement in perceived preparedness in all competency areas for both doctors and managers (supplementary file). The score improvement is likely to have been contributed to in part by other leadership and management experience gained by individuals over the 6-month pilot period. However, qualitative feedback collected from participants via our questionnaires and at our 6-month scheme celebration event suggests a significant benefit derived specifically from the Paired Learning scheme.

Both groups reported that after participating in Paired Learning they better understood the structure of each other's training and hierarchies, and how clinical and management decisions are made. They felt better able to work in partnership with the other group in managing patient services.

Doctors valued learning more about system and organization structure and felt that the programme has helped to give them more of a voice in the trust. The presence of junior doctors shadowing managers in their meetings has now inspired senior managers to look at inviting a junior doctor representative to regularly attend key management forums.

"Paired learning has given me access to the real world of NHS management ... I was surprised that I was actually able to make a valuable contribution to a meeting when I was shadowing" - Doctor

Managers benefited by gaining insight into challenges facing doctors and what their role exactly entails, and had first hand experience of other key issues whilst visiting clinical areas. The scheme has encouraged one senior manager to work with the education team to provide more leadership training for junior staff in 
the trust.

"When shadowing a PICU doctor I experienced first-hand the difficulties caused by flow issues, I was able to better articulate a case for the expansion of our long-term ventilation capacity" Manager

"Paired learning has been enlightening. Socialising with other managers and doctors has removed the element of being defined by one's title" - Manager

Workshop feedback was excellent with participants commenting on how useful they were, especially hearing both managers and doctors experiences and opinions. Group members particularly enjoyed the format of open frank discussion rather than lectures, and valued the opportunity to network with new colleagues, and to share knowledge and experiences in an informal setting.

Participants were surprised to find out the many similarities between the two groups and the common challenges that can affect their daily work activities.

Some pairs worked on business cases together, and others worked on NHS Change Day projects including the \#hellomynameis initiative which aimed to encourage staff members to introduce themselves to both patients and their colleagues.

All participants are planning on continuing some form of Paired Learning in the future.

See supplementary file: ds3903.jpg - "Graph of average evaluation scores pre and post-Paired Learning participation"

\section{Lessons and limitations}

Implementing this project at Birmingham Children's Hospital was relatively straightforward due to the support and enthusiasm shown to us by senior trust leaders, our local deanery, and trust education development team from the very beginning of the project. Their ongoing support and financial backing will help to make Paired Learning at Birmingham Children's Hospital a sustainable project for the future.

We are soon to commence our next project cycle, and have handed control of the trust Paired Learning scheme over to a new doctormanager pair, in order for Lydia and I to focus on developing the scheme at a regional and national level. We felt it was important for the scheme to continue to be run by a doctor and a manager as it reflects the spirit of the project. We have also joined up with the trust education development team who allow us access to training resources and speakers in order to support our workshops.

Following excellent feedback, we plan to continue with a similar workshop format, and have gathered plenty of ideas from participants for future workshop topics such as 'writing a business case' and 'developing a resilient team'.

Our only concern is that junior doctor turnover is much higher than that of managers, and we may run out of managers who are available and willing to participate. Also, after hearing about Paired
Learning, other professionals in our trust such as nurses and occupational therapists have expressed an interest in joining in, which is fantastic, but could further exacerbate the shortage of available managers.

We are going to monitor demand through the next phase of the project, and may consider limiting clinician participation through a selection process, or creating learning trios. Our idea for learning trios is that a manager could join with two doctors, or a doctor and a nurse. Not only would this expand the opportunities for individuals to shadow and learn from each other, but we hope that incorporating a wider skill mix would allow for successful and innovative group service improvement projects to be undertaken. We will repeat our evaluation next year, to assess the effect of any changes we have made to the project.

\section{Conclusion}

Paired Learning has proven to be an educationally valuable and popular addition to the experience offered to staff at Birmingham Children's Hospital. We are embedding Paired Learning in the ongoing trust educational programme, we have partnered with our LETB to extend Paired Learning region-wide, and we are even looking to develop the scheme nationally.

We hope that Paired Learning will further contribute to building the culture of team working, patient-centredness, mutual trust, and respect we are aiming to cultivate as an organisation. We also hope that the educational experience provided by the scheme will help to develop strong NHS leaders of the future.

We believe that if we can understand each other's role and work together then we have a real chance in meeting the challenges facing all of us in delivering excellent health care. Why don't you try Paired Learning for yourself? You don't even need a formal scheme, just introduce yourself to a manager and go from there.

\section{References}

1. http://bma.org.uk/about-the-bma/how-we-work/uk-and-natio nal-councils/bma-uk-council/council-chair-new-yearmessage-2014

2. Francis R. Report of the Mid Staffordshire NHS Foundation Trust Public Inquiry. February 2013. http://www.midstaffspublicinquiry.com/report

3. Darzi A. High Quality Care for All: NHS next stage review final report. London: Department of Health. 2008

4. Guthrie M. Engaging physicians in performance improvement. American Journal of Medical Quality, 2005; (20): 235-8

5. Klaber B, Lee J, Abraham R, Smith L, Lemer C. Paired Learning: A peer learning Leadership development initiative for managers and clinicians in the NHS. London: Imperial College Healthcare NHS Trust and NHS London.2012 http://www.londonleadingforhealth.nhs.uk/sites/default/files/ NHS\%20210sq.pdf

6. Paired Learning video http://youtu.be/GZ4N6sTuIRU 


\section{Declaration of interests}

nothing to declare

\section{Acknowledgements}

Lydia Salice, Dr Isobel Brookes, Dr Jeremy Tong, Alex Borg 